\title{
CORRECTED GYROCOMPASS SYNTHESIS AS A SYSTEM WITH CHANGEABLE STRUCTURE FOR AVIATION GRAVIMETRIC SYSTEM WITH PIEZOELECTRIC GRAVIMETER
}

\author{
Elena Nikolaevna BEZVESILNAYA ${ }^{1}$, Andriy Hennadiiovych TKACHUK ${ }^{2}$ \\ ${ }^{1}$ National Technical University of Ukraine "Kiev Polytechnic Institute", Peremogy Ave. 37, \\ Kyev 03056, Ukraine \\ ${ }^{2}$ Zhytomyr State Technological University, Chernyakhovskogo str. 103, Zhytomyr 10005, Ukraine \\ E-mail: andrew_tkachuk@i.ua (corresponding author)
}

Received 20 November 2013; accepted 20 August 201

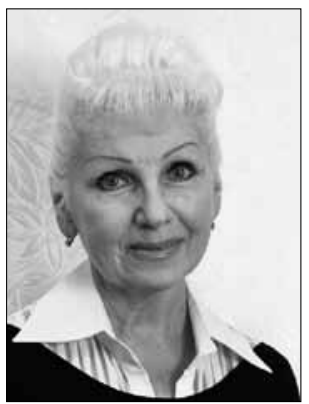

Elena Nikolaevna BEZVESILNAYA, Prof.

Education: sp. degree in gyroscopic instruments and navigation devices, National Technical University of Ukraine "Kiev Polytechnic Institute", Faculty of Instrumentation.

1972 to 1991: Candidate of Science, PhD in Science (Dr. techn Science), National Technical University of Ukraine "Kiev Polytechnic Institute", Faculty of Instrumentation; Academician of the Academy of Engineering Sciences in Ukraine, Member of New York Academy of Sciences, Honored Worker of Science in Ukraine.

Affiliations and functions: 1991 to present: professor, National Technical University of Ukraine "Kiev Polytechnic Institute", Department of Instrumentation.

Research interest: fundamental and applied problems of mechanics of gyroscopic navigation devices and moving objects, dynamics of air electromechanical systems.

Publications: 15 monographs, 9 books approved by the Ministry of Education and Science of Ukraine 30 patents, more than 100 scientific articles.

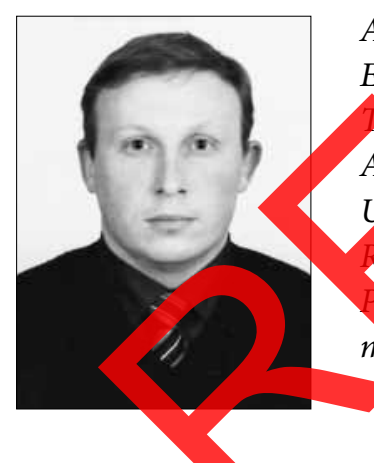

Andriy Hennadiiovych TKACHUK, PhD

Education. MSc degree in automated processes technological control, Zhytomyr State Pechnological University, Faculty of Information and Computer Technologies. Affiliations and functions: 2011 to 2014: PhD studies, Zhytomyr State Technological University, specialization: instruments and methods for measuring mechanical values. Research interest: gravimetric measurements and instruments, automatic control system. ublications: Author of 15 scientific articles and co-author of 21 conference papers, 2 monographs, 3 patents.

Abstract The present article introduces the development of a corrected gyrocompass with an automatic switch of the gyrocompass into the gyroazimuth mode. The issues of multi-criterion synthesis of the gyrocompass control loop have been studied. The necessity of temporary delay input (with selected values) under the previously mentioned switching is shown. On the basis of given requirements, we chose the parameters which provide the device with a switching-back into the gyrocompass mode under any initial gyrocompass deviations in the horizontal plane. An algorithm of gyrocompass ballistic deviation compensation due to the amendment generated by the special observing device, without recourse to external information regarding the ship's acceleration, was developed.

Keywords: gyrocompass, automatic switch, gravimetric system, piezoelectric gravimeter. 


\section{Introduction}

The accuracy requirements for the navigation parameters of basic aviation gravimetric system (AGS) subsystems with the resulting measurement accuracy of gravity anomalies with $1 \mathrm{mGal}$ are included in (Bezvesilna 2001): course -1.43 ang, width -0.5 ang, velocity -0.05 $\mathrm{m} / \mathrm{s}$, way $-1.5 \mathrm{~m}$, vertical velocity $-0.5 \cdot 10^{2} \mathrm{~m} / \mathrm{s}$, vertical acceleration $-1 \cdot 10^{-5} \mathrm{~m} / \mathrm{s}^{2}$.

A new piezoelectric gravimeter that is a part of the AGS is proposed in (Bezvesilna, Tkachuk 2014; Bezvesilnaya et al. 2013).

Usually, to determine the course on a plane, the gyropolukompas (GPK) is mainly used. However, the real errors of GPK are so great that definite accuracy cannot be obtained.

The purpose of this paper is to provide a corrected gyrocompass synthesis as a system with a changeable structure for the aviation gravimetric system with a piezoelectric gravimeter.

\section{The gyroscope compass}

Gyroscope compasses are the principal course-detecting devices for ships. The development of corrected gyrocompasses (CGC) on the basis of a biaxial platform with dynamically tuned gyroscopes has become one of the directions for developing a device that can meet the increased requirements for operational characteristics of navigation equipment. The main advantage of the $\mathrm{CG}$ is the possibility to change the structure and contre loop parameters depending on the device's operation mode. In particular, an effectiye method for decreasing the indication errors while the ship is maneuvering is to switch the device into the gyroazimuth mode, which can be done either manually or automatically. However, automatic switching into the gyroazimuth mode can result in cases when the device is not switched back to the gyrocompass mode. This isste has not been studied in full so far. The synthesis technique for a two-mode gyrocompass control loop remains a problem.

The given report presents the experience of developing such corrected gyrocompasses like CRUISE and SLAVUTICH by the Public Corporation "Kiev Plant of Automation named after G. Petrovsky" and "Marinex Company Limited" (Ukraine). The key aim of the studies was to maximally exhaust the possibilities for an autonomous change of the device operation mode.

Let's consider the peculiarities of CGC dynamics as a two-mode device (Malovichko, Kostitsyn 1992). Equations of motion are as follows:

$$
\begin{aligned}
& \dot{\alpha}-\omega_{\eta} \beta+\omega_{\xi} \gamma=-\dot{\beta} \gamma+r_{x} \delta ; \\
& \dot{\beta}+\omega_{\eta} \alpha-\omega_{\zeta} \gamma=\dot{\alpha} \gamma-r_{z} \delta ; \\
& \delta=\hat{O}(\partial)\left[\beta+g^{-1}\left(W_{\eta}-p^{2} \varphi \zeta_{0} \sin K\right)\right] \\
& J_{\eta} \ddot{\gamma}+f_{\gamma} \dot{\gamma}+m g l_{n} \gamma=m l_{n}\left(W_{\xi}-p^{2} \varphi \zeta_{0} \cos K\right)+ \\
& +f_{\gamma} p \varphi \cos K,
\end{aligned}
$$

where $\alpha, \beta$ indicate tilt angles of the main gyro axis from the direction to the North and from the horizon plane; $\delta$ - accelerometer filter output; $\gamma$ - platform tilt angle; $\varphi-$ roll angle; $r_{x}, r_{z}$ - coefficients; $\Phi(p)=\frac{1}{T p+1}-$ transfer function of the accelerometer filter, $T$ - filter time constant; $\omega_{\xi}, \omega_{\eta}-$ the East and North components of the Earth's rotation angular speed; $W_{\eta}$ - the North component of ship movement acceleration; $\zeta_{0}$ - the distance from the centre of gyro to the device installation place $J_{\eta}, f_{\gamma}, l_{n}$ - platform parameters; $g-$ gravitational acceleration.

To decrease the effect of movement acceleration on the device reading, while solving the inequality $|\delta| \geq|u|$, it is switched to the gyroazimuth mode $\left(r_{x}=0\right)$. Here, $u$ indicates a threshold value. If the inequality is $|\delta|<|u|$, the device is automatically switched to the gyrocompass mode. However, the accelerometer output signal can also take place as a result of initial device deviation in azimuth. Let's study this question in detail by analyzing the free motion on a fixed base. We shall neglect the influence of time constant, $T$ (that is admitted under the condition $\left.T<<r_{z}{ }^{-1}\right)$, and accept that $\beta(0)=0$, $\alpha(0)=\alpha_{0}$. The possible cases of movement are represented on a phase plane $\alpha-\beta$ (Fig. 1).

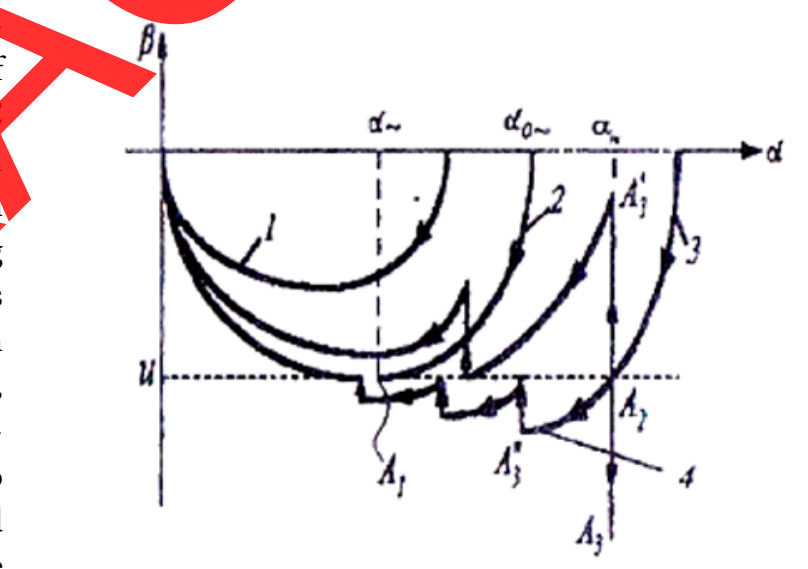

Fig. 1. The possible cases of movement

$$
\text { If } \alpha_{0}<\alpha_{0 \sim}=-u \frac{m_{2}}{\omega_{\eta}}\left(\frac{m_{1}}{m_{2}}\right)^{\frac{m_{1}}{m_{1}-m_{2}}} \text {, where } m_{1}, m_{2}
$$

indicate the roots of the characteristic equation, the device is constantly operating in the gyrocompass mode (curve 1). If $\alpha_{0}=\alpha_{0 \sim}$ (curve 2), then at a certain moment in time, $t_{\sim}$, the representing point will be in boundary position $A_{1}$, where $\dot{\alpha}=\dot{\beta}=0$. Since the movement is being stopped, there is no settling in the meridian.

Curve 3 corresponds to the movement in the case when $\alpha_{0}>\alpha_{0 \sim}$. Point $A_{2}$, is a transfer to the gyroazimuth mode, and further movement may be comfortably characterized by the diagram:

$$
\dot{\alpha}=0 \Rightarrow \omega_{\eta} \alpha_{\approx}+r_{z} u>0 \Rightarrow \dot{\beta}<0 \Rightarrow \beta \downarrow .
$$


The representing point will move down to the equilibrium position $A_{3}$. There is no settling in the meridian.

It is important to find out, when settling in the meridian will occur, under any initial conditions. One of the ways to execute this task is to increase coefficient $r_{z}$, when $\beta=u$. In this case we have $\omega_{\eta} \alpha_{\approx}+r_{z}^{\prime} u<0 \Rightarrow \dot{\beta}>0 \Rightarrow \beta \uparrow\left(r_{z}^{\prime}\right.$ indicates value $r_{z}$, in the gyroazimuth mode). For this, it is necessary to select coefficient $r_{z}^{\prime}$ from the condition $r_{z}^{\prime}>\left|\frac{\omega_{\eta} \alpha_{\approx}}{u}\right|$, or more accurately, $r_{z}^{\prime}>\left|\frac{\omega_{\eta} \sin \alpha_{\approx}}{u}\right|$. If $r_{z}^{\prime}>\frac{\omega_{\eta}}{u}$, then the CGC will be set in the meridian under any initial angle $\alpha_{0}$.

However, an increase of coefficient $r_{z}$ is not enough: it is necessary to also input a temporary delay $\tau_{g a-g c}$ switching the device from the gyroazimuth mode to the gyrocompass one. Then, during the delay time interval, the representing point will move upward in the gyroazimuth mode. In point $A_{3}{ }^{\prime}$ the device will be switched to the gyrocompass mode. This process is repeated further on. We can see that there is settling in the meridian.

Curve 4 represents the movement after the input of delay $\tau_{g c-g a}$, during the transfer from the gyrocompass mode to the gyroazimuth one. We see that settling in the meridian exists as well. It is efficient to input both delays

As shown in the results of modeling in figure 2 $\left(r_{x}=0.03 \mathrm{~s}^{-1} ; r_{z}=0.003 \mathrm{~s}^{-1}\right)$, coefficient $r_{z}$ was increased 10 times. Curve 1 corresponds to the delay of switching between the modes due to $\delta$ signal lag in the accelerometer filter with respect to angle $\beta$. It is accepted that $|u|<3$ angular minutes. Curve 2 corresponds to the input of an additional delay of $90 \mathrm{~s}$, while switching from the gyroazimuth mode to the gyrocompass one. Thus, due to an increase of coeflicient $r_{z}$ in the gyroazimuth mode and the input delay while changing the device operation mode, it is possible to provide its settling in the meridian under any initial ter

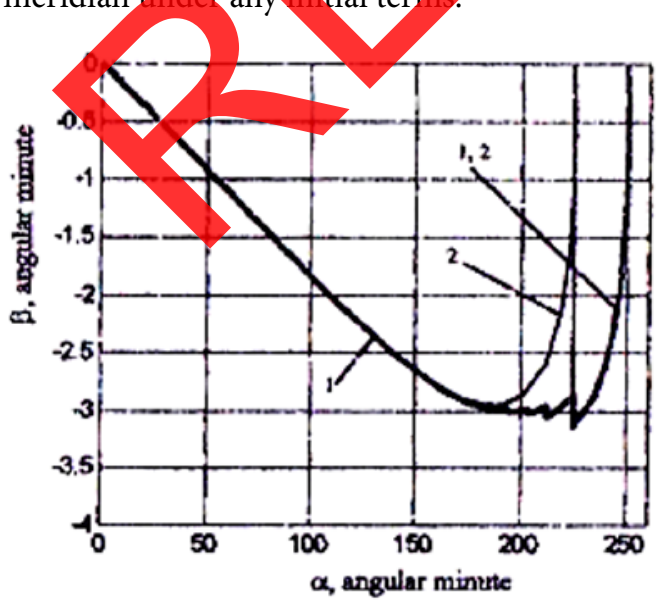

Fig. 2. Results of modeling
Let's consider the efficiency of switching the device to the gyroazimuth mode temporarily for a ship's maneuver. For this, we shall model equations of motion (1) with an acceleration $W_{\eta}=0.02 \mathrm{~m} / \mathrm{s}^{2}$ for $8 \mathrm{~min}$, which corresponds to speeding-up to 18 knots. We note, that while the ship is maneuvering, there is no need to increase coefficient $r_{z}$. This follows from the second equation of system (1), because with a = const, while increasing coefficient $r_{z}$, the main gyro axis tilts faster from the horizon plane. However, to make the device operative under any navigation conditions, the coefficient $r_{z}$ should always be increased while switching the device to the gyroazimuth mode. It should be taken into account that with a considerable increase of coefficient $r_{z}$ the decision nature can be changed: the process will become a light-extinction oscillating one. To exclude this, it is necessary to simultaneously decrease time constant $T$ (accepted $\left.T^{\prime}=5 s\right)$.

Figure 3 shows the modeling results. It is accepted that $|u|=3$ angular minutes, $\tau_{g a r}=90 \mathrm{~s}$. Curve 1 corresponds to switching to the gyroazimuth mode with an unchanged $r_{x}$; curve 2 -to $r_{z}$ increased 10 times; curve 3 - 0 increased $r_{x}$ and a decreased time constant $T$ to the value $T^{\prime}$. We see that in the third case there will be the least ballistic deviation. It is efficient that the gyrocompass is settled in the meridian much faster in the last

\section{case}

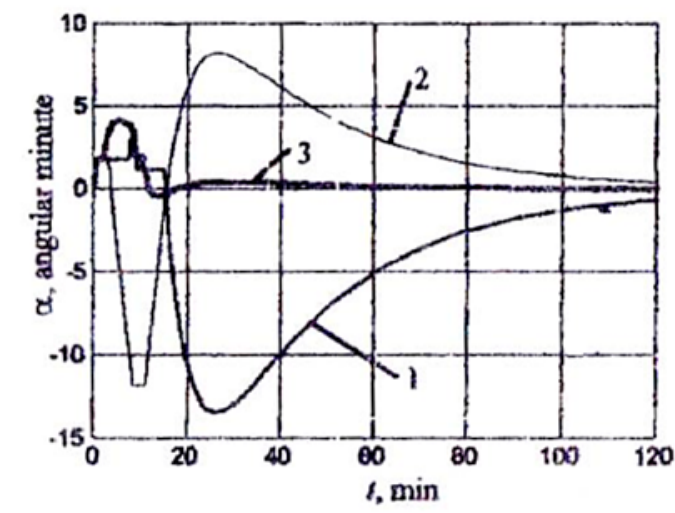

Fig. 3. The modeling results

Let's consider the CGC synthesis technique with a variable structure implemented in the CGC SLAVUTICH (Sternberg, Schwalm 2007). The task is: to choose such control loop parameters that the dynamic gyrocompass errors while maneuvering and rolling would not exceed the given values under the minimum time for the transient process.

Let's note that while rolling and settling into the meridian, the device must operate in the gyrocompass mode. Therefore, the posed task will be solved in the following way. Taking into account the requirement regarding accuracy while rolling, we shall impose constraints 
on the gyrocompass parameters $r_{x}, r_{z}$ and $T$. We select them, finally, minimizing the time for transient process. An admissible error while maneuvering will occur due the device's switching to the gyroazimuth mode. The following inequality can be derived from the expression for the gyrocompass' maximum error while rolling:

$$
\frac{r_{x}}{T} \leq \frac{2 \Delta K_{\max } \omega_{\eta} g \omega_{0}}{\zeta_{0} \varphi_{m}^{2} \omega_{k}^{2} d\left(1+\frac{\zeta_{0}}{g} \omega_{k}^{2}\right)},
$$

where $\Delta K_{\max }$ indicates admissible error while rolling.

The parameters will be selected during the mode of settling in the meridian on the basis of the root method (Sternberg, Schwalm 2007). According to this method the roots of a characteristic equation: $p^{n}+A_{1} \lambda_{0} p^{n-1}+A_{2} \lambda_{0}^{2} p^{n-2}+\ldots+\lambda_{0}^{n}=0$, should have an equal real part, but imaginary root parts should create an arithmetical progression the first member of which is equal to the arithmetical ratio. Here $\lambda_{0}$ indicates the middle geometrical root of a characteristic equation; $A_{1}, A_{2}$ - tabulated coefficients. Taking this into account, expressions $r_{z}=1.38 \lambda_{0}, \omega_{\eta} r_{x}=\lambda_{0}^{2}$ are achieved. Attention must also be payed to constraint $T<<r_{z}^{-1}$ that allows simplifying parameter synthesis in the gyroazimuth mode. Supposing, that $\varphi_{m}=20^{0} ; \omega_{k}=0.7 \mathrm{~s}^{-1} ; \zeta_{0}=6 \mathcal{M}$; $\omega_{0}=7 \mathrm{~s}^{-1} ; d=0.7 ; \Delta K_{\max }=1^{0} C \sec \varphi$, we shall that: $r_{z}=0.0012 \mathrm{~s}^{-1}, r_{x}=0.018 \mathrm{~s}^{-1}$ and $T=36 \mathrm{~s}$.

Figure 4 illustrates the plots of transient pro cesses, where curve 1 corresponds to the CGC CRUIS curve 2 - to the recommended paraneters. It can be seen that, due to the rational selection of the parameters, the time of the transient process is decreased considerably.

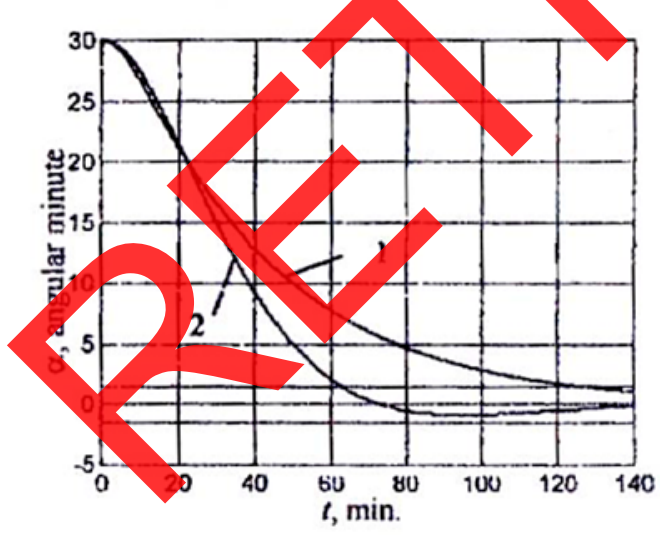

Fig. 4. The plots of transient processes

Let's consider the selection of parameters while maneuvering. The dependence of threshold value $u$ on temporary delay $\tau_{g c-g a}$ and maximum ballistic deviation is as follows:

$\left.|u|=\frac{\left|W_{\eta}\right|}{g} \mid 1-\left(1-\left|\frac{\Delta K_{6} g}{r_{x} \Delta V_{\eta}}\right|\right) e^{\frac{\tau_{g c-g a}}{T}}\right]$, where $\Delta V_{\eta}$ indicates the increment of the northern component of movement rate per maneuver time $t_{M}$.

Delay $\tau_{g c-g a}$ is required to avoid accidental device switching to the gyroazimuth mode while operating (towage, weighing the anchor, etc.). From the operation experience, it is accepted that $\tau_{g c-g a}=90 \mathrm{~s}$, which corresponds to $|u|=5$ angular minutes. Now we calculate $r_{z}^{\prime}>0.024 \mathrm{~s}^{-1}$ (with $r_{z}^{\prime}=0.03 \mathrm{~s}^{-1}$ ) from condition (1). According to inequality $T^{\prime} \leq \frac{1}{4 r_{z}{ }^{\prime}}$ we accept that $T^{\prime}=2 \mathrm{~s}$.

Delay $\tau_{g a-g c}$ will be calculated in the following way. The main gyro axis in the gyroazimuth mode is set quite rapidly in the equilibrium position, and signal $\delta$ becomes weaker than threshold value $u(|u|>|\delta|)$. Due to the input of this delay, it is possible not to admit the device to switch to the gyrocompass mode if the ship manetyer is still in progtess. Supposing that the maneuver time equals $1-3$ min, we will accept that $\tau_{g a-g c}=180$ s. Figure 5 represents the plots of ballistic deviations. Curve 1 corresponds to the CGC CRUISE and curve 2 - to the CGC with recommended paramters. It is evident that maximum deviation values are considerably lower than admissible values for $2^{\circ}$. The GC with recommended parameters results in less deviation and is settled in the meridian faster.

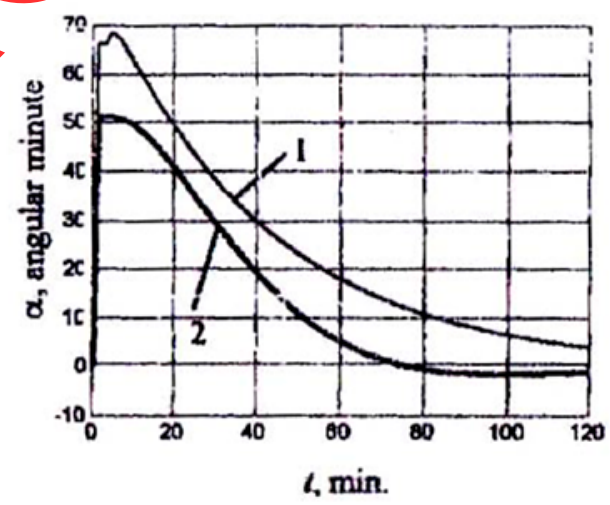

Fig. 5. The plots of ballistic deviations

CGC dynamic characteristics can be considerably improved by changing the structure of the accelerometer's filter. The performed analysis showed that it is efficient to use filters of an uneven degree. Using the root synthesis method, values $B_{1}=2086821 \mathrm{~s}^{3}, B_{2}=23184.6 \mathrm{~s}^{2}$, $B_{3}=218.6 \mathrm{~s}, r_{x}=0.077 \mathrm{~s}^{-1}, r_{z}=0.0030 \mathrm{~s}^{-1}$ have been received for a third degree filter with the transfer function $\Phi(p)=\left(B_{1} p^{3}+B_{2} p^{2}+B_{3} p+1\right)^{-1}$ for a settling time of $30 \mathrm{~min}$. The transient process plot is illustrated in figure 6 by curve 3 . Curves 1 and 2 correspond to analogous filters of the $1^{\text {st }}$ and $2^{\text {nd }}$ degrees. We can see that the transient process time can be essentially decreased.

It is obvious that the third stage filter allows decreasing the error while rolling considerably: in comparison with CGC CRUISE error, it was decreased 900 
times. This allows installing the device at a considerable distance from the center of rolling (30 $\mathrm{m}$ and more).

Error reduction on the basis of information estimation with the help of observing devices is one of the effective methods to increase the accuracy of navigation systems. Let us take under consideration the application of this method to decrease gyrocompass ballistic deviations (Malovichko, Kostitsyn 1992).

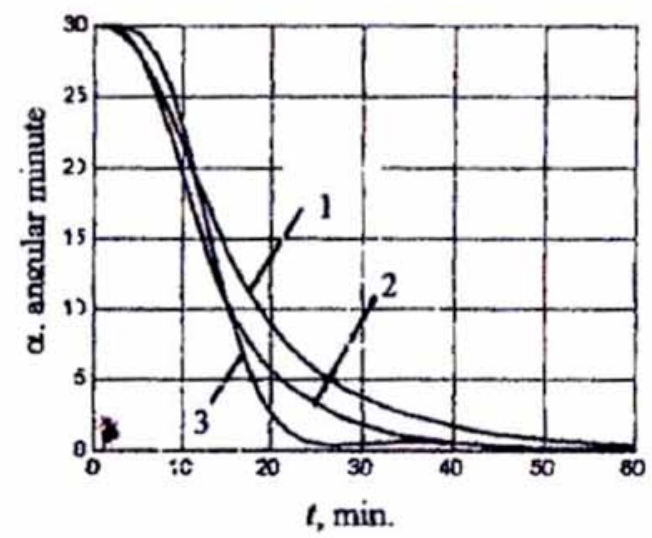

Fig. 6. The transient process plot

We shall take the following equations as the gyrocompass model:

$$
\begin{aligned}
& \dot{\alpha}_{m}-r_{x m} \delta_{m}=k_{1}\left(\delta-\delta_{m}\right) ; \\
& \dot{\beta}_{m}+\omega_{\eta} \alpha_{m}+r_{z m}=k_{2}\left(\delta-\delta_{m}\right) ; \\
& T_{m} \dot{\delta}_{m}+\delta_{m}-\beta_{m}=0,
\end{aligned}
$$
where $\alpha_{m}, \beta_{m}$ and $\delta_{m}$ indicate the model variables corresponding to real variables; $r_{x m}, r_{z m}, T_{m}$ - model parameters; $k, k_{2}$ - coefficients.

Indicating that $\Delta \alpha=\alpha-\alpha_{m}, \quad \Delta \beta=\beta-\beta_{m}$, $\Delta \delta=\delta-\delta_{m}$ and supposing that $r_{x m}=r_{x}, r_{z m}=r_{z}$, we shall get the following system of equations for calculating estimation error

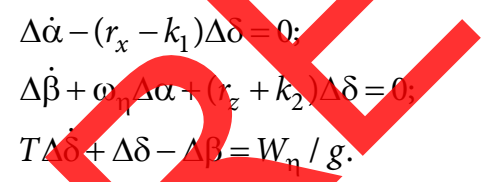

Moving on to the question of coefficient selection of $k, k_{2}$, for the time of the maneuver, we will accept that $k_{1}=r_{x}, k_{2}=r_{z}{ }^{\prime} r_{z}$. In this case, the equations of errors (5) become:

$$
\begin{aligned}
& \Delta \dot{\alpha}=0 ; \\
& \Delta \dot{\beta}+\omega_{\eta} \Delta \alpha+\left(r_{z}+k_{2}\right) \Delta \delta=0 ; \\
& T \Delta \dot{\delta}+\Delta \delta-\Delta \beta=W_{\eta} / g .
\end{aligned}
$$

It is evident that estimation error $\Delta \alpha$ does not depend on the acceleration value and is defined only by its initial value. As physically the gyrocompass is not switched to the gyroazimuth mode, we shall call this concept "the mode of analytical switching to the gyroazimuth mode".
After maneuver termination, we accept that $k_{1}=$ $k_{2}=0$. In this case, the equations of errors with the accuracy up to designations will meet the equations of the gyrocompass free motion.

To change the coefficients of the observing device, it is necessary to have the information about either the availability or absence of ship movement acceleration, which can be obtained by analysing the output signal level of the horizon indicator.

The modeling of the gyrocompass dynamics with an observing device while the ship is moving with an acceleration $W_{\eta}=0.172 \mathrm{~m} / \mathrm{s}^{2}$ within $1 \mathrm{~min}$., which corresponds to the speeding-up to 20 knots, was carried out to check the efficiency of the proposed ballistic deviation compensation algorithm.

In figure 7 , curve 1 corresponds to ballistic deviation under physical switching of the gyrocompass to the gyroazimuth mode and curve 2 to analytical switching. The estimation error of ballistic deviation of the observing device, under permanent operation of the gyrocompass in the main mode (curve 2), is slightly different from ballistic deviation under physical device switching to the gyroazimuth mode.

While using the observing device, the apparatus is operating in the gyrocompass mode all the time; therefore, it is not a problem for the main axis to set in the meridian under large initial deviation angles. There is, ence, no need to increase coefficient $r_{z}$ for the time of operation in the gyroazimuth mode (curve 3 ).

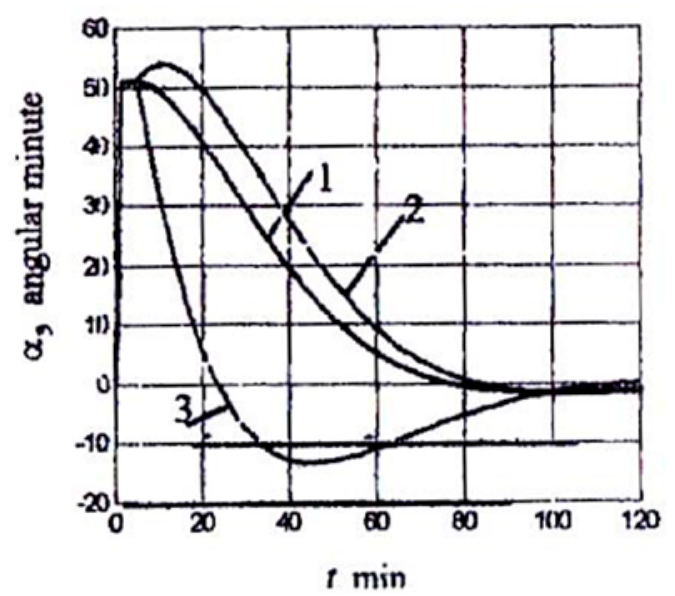

Fig. 7. Ballistic deviation under physical switching of the gyrocompass to the gyroazimuth mode

The matters of gyrocompass stabilization system projection will be discussed further (Bohnenberger 1817). The structural scheme of the gyrocompass' stabilization system projection is shown in figure 8 , where $\mathrm{x}$ and $\sigma$ indicate the input and output variables; $m$ - disturbance. To exclude static errors, let us regard the stabilization system as a static one according to both input 
and disturbance. The transfer functions of the control object $\Phi_{0}(p)$ and regulator $\Phi_{\phi}(p)$ will be as follows

$$
\Phi_{0}(p)=\frac{K_{0}}{p(T p+1)}, \Phi_{\phi}(p)=\frac{1}{p} K N(p),
$$

where $K_{0}, T$ and $K$ are parameters; $N(o)=1$.

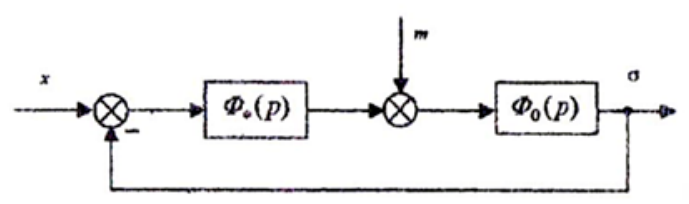

Fig. 8. The gyrocompass' stabilization system projection

Usually, the maximum value of the output variable is determined according to disturbance: it is essential to define the structure and parameters of the regulator transfer function, where the extreme value of variable $\sigma(t)$, as the reaction on disturbance $m$, equals the given value. The given parameter is also an index of oscillation $M$ of the closed-loop system (with respect to input $x$ ). Let us transform the structural scheme to the shape represented in figure 9. Here $\Phi_{p}(p)=\Phi_{0}(p) \Phi_{\phi}(p)$ : indicating the transfer function of the open-loop system. The transfer function $N(p)$ is chosen so that extreme values of $u_{m}, \sigma_{m}$ variables $u(t)$ and $\sigma(t)$ are equal.

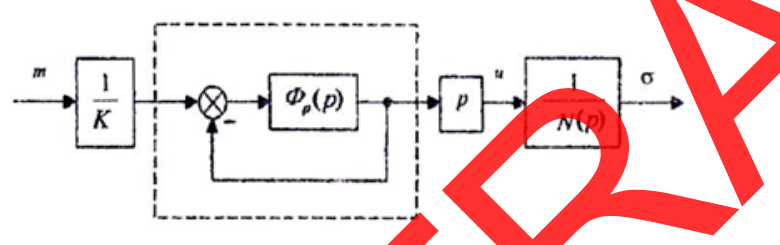

Fig. 9. The structural scheme to the shape

The transfer function of the open-loop etalon system is accepted as follows:

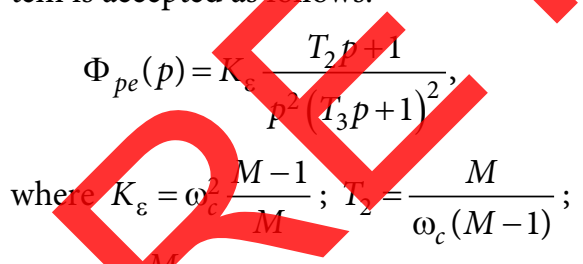

$T_{3}=\frac{M}{2 \omega_{c}(M-1)} ; \omega_{c}$ indicates cutoff frequency

(Bohnenberger 1817).

The extreme value of variable $u(t)$ of the etalon system is equal to:

$$
u_{e m}=\omega_{c} \rho \frac{m}{K}
$$

where $\rho=\lambda \sqrt{\frac{M-1}{M}} ; \lambda=1.7 ; 1.49 ; 1.4$ for

$M=1.3 ; 1.5 ; 1.7$ respectivelly.

Supposing that $\sigma_{m}=u_{m}$, we get the following expression for the coefficient of regulator amplification:

$$
K=\frac{m \rho \omega_{c}}{\sigma_{m}} .
$$

The transfer function $\mathrm{N}(p)$ is chosen as follows:

$$
N(p)=\frac{\left(T_{11} p+1\right)\left(T_{12} p+1\right)\left(T_{2} p+1\right)}{\left(T_{21} p+1\right)\left(T_{3} p+1\right)^{2}},
$$

and the transfer function $\Phi_{p}(p)$ is recorded in the following way:

$$
\Phi_{p}(p)=\Phi_{0}(p) \Phi_{k}(p)=E(p) \Phi_{p e}(p),
$$

where $E(p)=R \frac{\left(T_{11} p+1\right)\left(T_{12} p+1\right)}{\left(T_{21} p+1\right)(T p+1)}$;

$R=\frac{K K_{0}}{K_{\varepsilon}}=\frac{m K_{0} \lambda^{2}}{\sigma_{m} \omega_{c} \rho}$.

The transfer function parameters $\mathrm{E}(p)$ are chosen so the expressions $\left(T_{11} \omega_{c}\right)^{2}>>1 ;\left(T_{12} \omega_{c}\right)^{2}>>1 ;\left(T_{21} \omega_{c}\right)^{2}>>1 ;\left|E\left(i \omega_{c}\right)=1\right|$ are done in the area of cutoff frequency. In this case:

$$
R=\frac{T_{21} T}{T_{11} T_{12}}
$$

Let us now take into account the requirement that $\sigma_{m}=u_{m}$, which is defined by the parameters of transfer function $N(p)$. According to the results of the analysis, he following expression is obtained:

$$
\frac{T_{21}}{T_{11} T_{12}}=\frac{\omega_{\tilde{n}}}{\sqrt{\rho}} .
$$

By equalizing two received expressions for coefficient $R$, taking into account expression (13), the cutoff frequency is obtained as:

$$
\omega_{\tilde{n}}=\frac{\lambda}{\sqrt[4]{\rho}} \sqrt{\frac{m K_{0}}{\sigma_{m} T}} .
$$

Time constants $\mathrm{T}_{11}$ and $\mathrm{T}_{12}$ should be chosen of approximately one order higher than time constant $T_{2}$.

As an example, let us consider the system of azimuthal stabilization with these parameters: $\sigma_{m}=2$ angular min.; $m=0.04 \mathrm{~N} \cdot \mathrm{m} ; T=2 \mathrm{~s} ; K_{0}=500(\mathrm{~N}-\mathrm{m}-\mathrm{s})^{-1}$, $\mathrm{M}=1.3 ; 1.5 ; 1.7$. The results of modeling are shown in figure 10 .
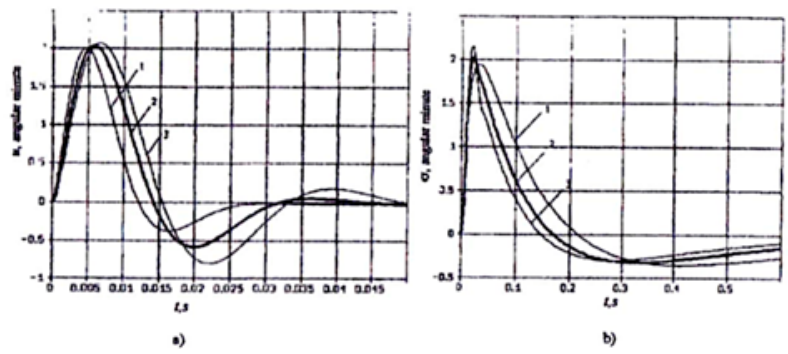

Fig. 10. The results of modeling

It is evident from the figures that the proposed method allows synthesizing a static stabilization systems rather simply and with high accuracy.

The experience of the CGC CRUISE operation confirmed the efficiency of the proposed scheme and technical decisions and revealed that it is possible to further improve this type of gyrocompass. 


\section{Conclusions}

The corrected gyrocompass synthesis as a system with a changeable structure for the aviation gravimetric system with a piezoelectric gravimeter is provided in this paper. The experience of developing such corrected gyrocompasses like CRUISE and SLAVUTICH by the Public Corporation "Kiev Plant of Automation named after G. Petrovsky" and "Marinex Company Limited" (Ukraine) are presented.

Modeling was conducted and found that due to an increase of coefficient $r_{z}$ in the gyroazimuth mode and the input of delay while changing the device operation mode, it is possible to provide its settling in the meridian under any initial terms; CGC dynamic characteristics can be considerably improved by changing the structure of the accelerometer's filter; error reduction on the basis of information estimation with the help of observing devices is one of the effective methods to increase the accuracy of navigation systems.

\section{References}

Bezvesilna, O. M. 2001. Vysmirjuvannja pryskoren. Kyiv: Lybid. (in Ukrainian).

Bezvesilna, O. M.; Tkachuk, A. G. 2014. Naukovo-teoretychni doslidzhennja novogo p'jezoelektrychnogo gravimetra avtomatyzovanoi' aviacijnoi' gravimetrychnoi' systemy ekstremal'nyh umovah $z$ vykorystannjam nejronnyh merezh Zhytomyr: ZhDTU. (in Russian).

Bezvesilnaya, E.; Tkachuk, A.; Ostapchuk, A., et al. 2013 Angle measurement device for posting the sensitivity axis of aviation gravimeter, The Advanced Science Joumal (12): 31-37.

Bohnenberger, J. G. F. 1817. Beschreibung Einer Maschine zur Erläuterung der Gesetze der Undrehung der Erde um ihre Axe, und der Veränderung der Lage der Letzteren [Description of a machine forthe explanation of the laws of rotation of the Earth around its axis, and of the change of the orientation of the latter], Tübinger Blätter für Naturwissenschaften und Arzneikunde 3: $72-83$. (in German).

Malovichko, A. K.; Kostitsyn, V 1. 1992. Gravirazvedka. Moskva: Nedra. (in Russian).

Sternberg, H. Schwalm, C. 2007 Qualification process for MEMS gyroscopes for the use in navigation systems, in Proceedings of International Society for Photogrammetry and Remote Sensing, 2007, Hamburg, Germany. 